\title{
CYCLES ON ABELIAN VARIETIES ${ }^{1}$
}

\section{ARTHUR MATTUCK}

The object of this paper is to study the algebraic homology with rational coefficients of the general complex abelian variety. By this we mean an $n$-dimensional complex torus whose period matrix satisfies no relations other than the Riemann relations. It turns out that despite the richness of the general abelian variety in topological cycles, algebraically it is just like complex projective space: in each complex dimension the only homology classes represented by algebraic subvarieties are the lone homology class of the linear section of that dimension and its rational multiples. Further, the only homology classes of the torus "lying on" these subvarieties are those arising in the obvious way-intersection of the subvarieties with the homology classes of the torus.

The method of proof is to show somewhat explicitly that Hodge's necessary conditions that a cycle lie on a subvariety are in this case also sufficient. Whether they are in general is of course unknown. It turns out that a little-known paper of Comessatti [3] is closely connected with this result, so closely in fact that the present paper should essentially be regarded as a resuscitation, expansion, and interpretation of Comessatti's work.

1. A topological $p$-cycle on an algebraic variety $V^{n}$ of dimension $n$ is said by Hodge [5] to be of rank $r$ if it is homologous to a cycle lying in an algebraic subvariety of complex dimension $p-r$. For such a cycle $\sigma$,

$$
\int_{\sigma} \phi^{(p-i, i)}=0 \quad i=(p-n)^{+}, \cdots, r-1,
$$

for every closed $p$-form $\phi$ of type $(p-i, i)$; here $(p-n)^{+}$means $\max (p-n, 0)$. Indeed, since $\phi$ is closed, this integral is the same as one taken over the cycle lying in the subvariety. But $\phi$ restricted to any component of the subvariety must be identically zero, since a variety of complex dimension $p-r$ cannot support a differential containing $p-i d z$ 's, where $p-i>p-r$.

Supposing now the variety to be endowed with its Kahler metric,

Received by the editors July 14, 1957.

1 This research was supported in part by the United States Air Force under Contract No. AF 18(603)-91, monitored by the Air Force Office of Scientific Research, Air Research and Development Command. 
in particular the harmonic $(p-i, i)$ forms must have zero periods on $\sigma$, and this is Hodge's condition. Conversely, if the harmonic $(p-i, i)$ forms have zero period on $\sigma$, then so does every closed $(p-i, i)$ form $\phi$, since by the Kahler property, $H \phi$ is also of type $(p-i, i)$ and $\phi \sim H \phi$, so that they have the same periods. Thus the Hodge condition depends really only on the complex structure and not on the choice of metric.

We will deal here only with a weak form of the notion of the "rank" of a cycle: a p-cycle is of rational rank $r$ if some integral multiple of it is of rank $r$. In other words, we abandon integral coefficients for rational coefficients; the methods permit estimation of the integral multipliers that suffice, but not whether they are best possible. We shall show that on the general abelian variety $V^{n}$, if a $p$-cycle $\sigma$ satisfies the appropriate Hodge condition, then it is indeed of rational rank $r$ and in the following strong way: if $E^{m}$ denotes the general linear section of $V$ of complex dimension $m$, then $\sigma \sim E^{p-r} \cdot \tau$ for a suitable rational cycle $\tau$. In other words, $\sigma$ is actually homologous to a cycle in $E^{p-r}$ produced by intersection.

If a p-cycle is "algebraic"- that is, is homologous to the fundamental cycle of an algebraic subvariety-then it is a $2 r$-cycle of rank $r$. By the above, it follows that the only algebraic nonzero rational homology classes on the general abelian variety are those represented by the linear sections of the various dimensions and their rational multiples. (In dimension $n-1$, this is well-known.) Further, the only rational cycles of the variety lying on these subvarieties are, up to homology, those arising in the trivial way by intersection. It follows then (see corollary) that the dimension of the space of basic $p$-cycles of rank $r$ is $C_{2 n, p-2 r}$.

Notation. By $I$ (or $J$ or $K$ ) we always mean a sequence of integers $i_{1} i_{2} \cdots i_{p}$ selected from the integers $1,2, \cdots, 2 n$ and arranged in increasing order.

$I-i_{k}$ means the sequence $I$ with $i_{k}$ removed.

$I^{\prime}$ denotes the complementary sequence: $1,2, \cdots, 2 n$ with $i_{1}, \cdots, i_{p}$ removed. Thus $I I^{\prime}$ is a permutation of $1,2, \cdots, 2 n$ and we let $\epsilon_{I I^{\prime}}$ denote the sign of this permutation.

$|I|$ denotes the length of $I$. Here for example $|I|=p,\left|I^{\prime}\right|=2 n-p$, $\left|I-i_{k}\right|=p-1$.

$d x_{I}$ stands for the differential $d x_{i_{1}} d x_{i_{2}} \cdots d x_{i_{p}}$.

Given a $2 n \times 2 n$ matrix, the unsigned determinant minor formed from the rows $i_{1}, \cdots, i_{p}$ and the columns $j_{1}, \cdots, j_{p}$ will be denoted by $[I, J]$. The corresponding signed minor will be written $\{I, J\}$. Explicitly therefore 


$$
\{I, J\}=\epsilon_{I I^{\prime}} \epsilon_{J J^{\prime}}[I, J] \text {. }
$$

2. We summarize now the needed facts about an $n$-dimensional complex abelian variety $V^{n}$, bianalytically represented in the usual way as a torus obtained from the $2 n$ real-independent vectors $v_{1}, \cdots, v_{2 n}$ in complex affine $n$-space $C^{n}$. Let the coordinates in $C^{n}$ be $z_{1}, \cdots, z_{n}$ and set $z_{i+n}=\bar{z}_{i}$. The Riemann matrix $M$ associated with the torus has for its $2 n$ columns the vectors $v_{i}$; below $M$ we place $\bar{M}$, the matrix whose entries are the conjugates of the entries of $M$, and we call the new $2 n \times 2 n$ matrix thus resulting $\mathfrak{M}$.

The homology ring of the complex torus is a Grassmann ring on $2 n$ generators. A typical basic $p$-cycle is given by selecting $p$ of the vectors, $v_{i_{1}}, \cdots, v_{i_{p}}$, orienting them by writing the $i_{k}$ in increasing order, and taking the real-linear subspace of the torus that they span: the set of vectors $t_{1} v_{i_{1}}+\cdots+t_{p} v_{i_{p}}, 0 \leqq t_{k}<1$. We denote this cycle by $\left\langle i_{1}, \cdots, i_{p}\right\rangle$, or simply $\langle I\rangle$ for short, so that a set of basic $p$-cycles which represent the rational homology classes is

$$
\sum a_{I}\langle I\rangle, a_{I} \text { rational numbers, }|I|=p .
$$

If we take the metric on the torus induced from the Euclidean metric on $C^{n}$, the complex harmonic $p$-forms on the torus are just the translation-invariant ones: $\sum c_{K} d z_{K}$, where $|K|=p$, and the $c_{K}$ are complex numbers. In this notation, a basis for the space of harmonic $(p-i, i)$ forms would be the forms $d z_{R S}$, where $|R|=p-i$, $|S|=i$, and $1 \leqq r_{\alpha} \leqq n, n+1 \leqq s_{\beta} \leqq 2 n$. We shall say in this case for convenience that the symbol $K=R S$ is of type $(p-i, i)$.

Then by the usual formula in analytic geometry for the volume of a $p$-dimensional parallelopiped, we integrate harmonic $p$-forms on basic $p$-cycles according to the rule

$$
\int_{\langle I\rangle} d z_{K}=[K, I] ; \quad|I|=|K|=p .
$$

Thus Hodge's necessary condition $(\$ 1)$ that the $p$-cycle $\sum a_{I}\langle I\rangle$ on the torus be of rank $r$ reads explicitly: for each $K$ of type $(p-i, i)$, where $i=(p-n)^{+}, \cdots, r-1$, we have the relation $\sum_{I} a_{I}[K, I]=0$, and our object is to prove it sufficient for the general abelian variety.

To get rid of the cycles completely, we translate everything into forms by the Poincaré-de Rham duality theory. To do this, first let $E$ be a given divisor on the abelian variety. By a complex linear transformation of the $z_{i}$ and a unimodular transformation of the vectors $v_{i}$ (whose names we shall not change, however), the Riemann matrix may be put into normal form relative to $E$, namely 


$$
\left(\frac{\delta_{i j}}{d_{i}}, A\right), \quad d_{i} \text { integers, } d_{i} \mid d_{i+1} .
$$

Here $A$ is a symmetric matrix whose imaginary part is positive definite, and $d_{i}$ is the intersection number $i(E \cdot\langle i, i+n\rangle)$ of $E$ with the $i$ th 2 -cycle. Conversely, such a matrix defines an abelian variety bearing a divisor $E$ with the indicated intersection property [1].

We now let the basis vectors $v_{1}, \cdots, v_{2 n}$ in $C^{n}$ which make up the columns of the matrix in the above normal form be the unit vectors in a real coordinate system $x_{1}, \cdots, x_{2 n}$. By the de Rham-Hodge theorem, we may identify the real harmonic $p$-forms $\sum a_{I} d x_{I}$ with the real $p$-cohomology classes. The Poincare dual of the $p$-cycle $\langle I\rangle$ is the harmonic $2 n-p$ form $\phi$ defined by the relation

$$
\int_{\langle I\rangle} \psi=\int_{V} \phi \wedge \psi, \text { all } p \text {-forms } \psi
$$

If we write $\mathfrak{D}$ for the Poincare duality operator, then since $\int_{\langle I\rangle} d x_{I}=1$, it is easy to see that

$$
\mathfrak{D}\langle I\rangle=\epsilon_{I^{\prime} I} d x_{I^{\prime}} .
$$

Further, the intersection formula $i(E \cdot\langle i, i+n\rangle)=d_{i}$ establishes that if we let $\omega=\mathfrak{D} E$, then

$$
\omega=\sum_{1}^{n} d_{i} d x_{i} d x_{i+n}
$$

It follows that if $E^{m}$ is the $m$-dimensional algebraic cycle $E \cdot E \cdots \cdots E$ $(n-m$ factors $)$, then

$$
\mathfrak{D}\left(E^{m} \cdot \sigma\right)=\omega^{n-m} \cdot \mathfrak{D} \sigma .
$$

3. We now suppose the abelian variety to be general, by which we mean that in the normal form representation of its Riemann matrix relative to some divisor $E$, if the matrix $A$ has as entries $a_{i j}=a_{i j}^{\prime}$ $+i a_{i j}^{\prime \prime}$, then the $a_{i j}^{\prime}$ and $a_{i j}^{\prime \prime}$ should be $n(n+1)$ independent real transcendental numbers. Under these circumstances, one knows that the normal form is fixed; changing the coordinates or the vectors cannot lead to a normal form with different $d_{i}$ 's or a different matrix (except to multiply all the $d_{i}$ by a constant factor). $E$ is then algebraically equivalent to some multiple of a hyperplane section on the variety, thought of as embedded in a suitable projective space by means of $\theta$-functions.

The theorem of $\S 1$ is then explicitly 
Theorem $(n, p, r)$. On the general abelian variety $V^{n}$ let $\sigma=\sum a_{J}\langle J\rangle$ be a basic rational $p$-cycle. If for each $I$ of type $(p-i, i)$, where $i$ $=(p-n)^{+}, \cdots, r-1$, we have the relation

$$
\sum_{J} a_{J}[I, J]=0
$$

among the minors of the "doubled" Riemann matrix $\mathfrak{M}$, then $\sigma \sim E^{p-r} \cdot \tau$, where $\tau$ is a rational cycle.

Let us apply the Poincare duality operator $\mathfrak{D}$ to the relation $\sigma \sim E^{p-r} \cdot \tau$, by using the formulas of $\S 2$. It becomes

$$
\sum a_{J} \epsilon_{J^{\prime} J} d x_{J^{\prime}}=\omega^{n-p+r} \phi
$$

where $\phi$ is a harmonic form in the $d x$ 's with rational coefficients, since we know that $\mathfrak{D}$ carries rational cycles into such forms. Use this as the new conclusion of theorem $(n, p, r)$, and put $K=J^{\prime}$ and $a_{K}$ $=a_{J} \epsilon_{J^{\prime} J}$; then it is easy to see that the theorem takes the equivalent form

TheOREM $(n, p, r)$. Let $\sum a_{K} d x_{K}$ be a $2 n-p$ form with rational coefficients $a_{K}$. If for each I of type $(p-i, i)$, where $i=(p-n)^{+}, \cdots$, $r-1$, we have the relation

$$
\sum_{K} a_{K}\left\{I, K^{\prime}\right\}=0
$$

among the signed minors of the matrix $\mathfrak{M}$, then

$$
\sum a_{K} d x_{K}=\omega^{n-p+r} \phi
$$

where $\phi$ is a form in the dx's with constant rational coefficients.

In this theorem, the admissible ranges on $n, p, r$ we will take to be

$$
n \geqq 1, \quad 0 \leqq p \leqq 2 n, \quad(p-n)^{+} \leqq r \leqq \frac{p}{2}+1 .
$$

For $p=0, n$ the theorem is trivial, making the obvious interpretations.

For $r=(p-n)^{+}$, the statement has no meaning; we admit this value of $r$ only for formal reasons and take the corresponding theorem $\left(n, p,(p-n)^{+}\right)$to be the vacuously true proposition.

For $r=[p / 2]+1$ the theorem is also true. Namely, $(n, p,[p / 2]+1)$ says that the harmonic integrals of type $(p-i, i), i=(p-n)^{+}, \cdots$, $[p / 2]$ have zero periods on the cycle $\sum a_{J}\langle J\rangle$. By conjugation, the integrals of the remaining types must also have zero periods. Therefore $\sum a_{J}\langle J\rangle \sim 0$, so that all $a_{J}=0$ and there is nothing to prove. 
Since Hodge's condition is a necessary one, the converse of the theorem is known to be true, and we shall use it at several points in the argument.

We shall prove theorem $(n, p, r)$ by induction, showing that the inductive hypotheses $(n-1, p, r),(n-1, p-1, r)$, and $(n-1, p-2, r)$ together imply $(n, p, r)$. Those cases $(n, p, r)$ for which one of the induction hypotheses is meaningless because the dimensions do not lie within the admissible ranges are the starting points for the induction. Inspection shows that all of them are one of the above two types: $\left(n, p,(p-n)^{+}\right)$, or $(n, p,[p / 2]+1)$, both of which are by the above conventions true.

4. What Comessatti proves (Teorema di Completezza) is essentially the case $(n, p, 1), p \leqq n$, of the above, by the same sort of induction employed here. He also phrases his theorem in terms of differential forms, his being related to the above ones by the star operation. The forms are introduced as a symbolic representation of linear complexes, and his proof is geometric, phrased as a study of the "total spaces" of a linear complex. But earlier in the paper the theorem is interpreted as a result about lower-dimensional singularity indices of Riemann matrices.

5. The key to the inductive proof we will give consists in simply this: if the $n \times 2 n$ Riemann matrix $M$ in normal form represents a general abelian variety $V^{n}$, then by suppressing the $n$th row and the $n$th and $2 n$th columns of $M$, we are left with an $(n-1) \times(2 n-2)$ matrix which is again immediately seen to be the Riemann matrix in normal form of a general abelian variety $V_{0}^{n-1}$. The differential form on $V_{0}$ which is the dual of the hyperplane section we denote by $\omega_{0}$; it is $\sum_{1}^{n-1} d_{i} d x_{i} d x_{i+n}$. The natural projection mapping of $V$ onto $V_{0}$ is not complex analytic, only real analytic. Further, the argument depends heavily on the explicit form of $\mathfrak{M}$, and this should be kept in mind in all that follows.

We start then with the $2 n-p$ form $\sum a_{K} d x_{K}$, and we break it up into the sum of four forms:

$$
\sum a_{K} d x_{K}=d x_{n} \phi_{1}+d x_{2 n} \phi_{2}+d x_{n} d x_{2 n} \phi_{3}+\phi_{4}
$$

where none of the $\phi_{i}$ contains either $d x_{n}$ or $d x_{2 n}$. We will show that each of the three forms $d x_{n} \phi_{1}, d x_{2 n} \phi_{2}$, and $d x_{n} d x_{2 n} \phi_{3}+\phi_{4}$ can be written as $\omega^{n-p+r} \psi_{i}$.

PART A. Suppose we have shown that $\phi_{1}$ and $\phi_{2}$ both satisfy the hypothesis of $(n-1, p-1, r)$. Then by the induction, it follows that $\phi_{i}=\omega_{0}^{n-p+r} \psi_{i}, \quad i=1,2$. But, putting $\omega_{n}=d x_{n} d x_{2 n}$ and noting that $\omega=\omega_{0}+d_{n} \omega_{n}$, we have 


$$
\omega^{n-p+r}=\omega_{0}^{n-p+r}+(n-p+r) d_{n} \omega_{n} \omega_{0}^{n-p+r-1}
$$

from which it follows that $d x_{n} \omega_{0}^{n-p+r}=d x_{n} \omega^{n-p+r}$ and $d x_{2 n} \omega_{0}^{n-p+r}$ $=d x_{2 n} \omega^{n-p+r}$. Thus we have

$$
d x_{n} \phi_{1}=\omega^{n-p+r}\left(d x_{n} \psi_{1}\right) \quad \text { and } \quad d x_{2 n} \phi_{2}=\omega^{n-p+r}\left(d x_{2 n} \psi_{2}\right)
$$

as promised.

Therefore we are assuming that $\sum a_{K} d x_{K}$ satisfies the hypothesis of theorem $(n, p, r)$ : for each $I$ of type $(p-i, i)$, where $i=(p-n)^{+}$, $\cdots, r-1$, we have

$$
S_{I} \equiv \sum_{K} a_{K}\left\{I, K^{\prime}\right\}=0
$$

and we have to show $\phi_{1}$ and $\phi_{2}$ satisfy the hypothesis of theorem $(n-1, p-1, r)$.

To do this, we need only look at those relations $S_{I}=0$ for which $n \in I, 2 n \notin I$, so just consider these. When the minors occurring in $S_{I}$ are expanded out, the relation $S_{I}=0$ becomes an identity among transcendental elements. In particular, therefore,

(i) all terms involving $a_{n, 2 n}$ make up a separately vanishing sum $T_{I}$, and it turns out that the equations which express that $T_{I}=0$ are precisely the induction hypothesis $(n-1, p-1, r)$ for $\phi_{1}$;

(ii) all terms not involving any transcendental elements of row $n$ or column $2 n$ make up a separately vanishing sum $U_{I}$, and the equations expressing that $U_{I}=0$ are the induction hypothesis for $\phi_{2}$.

To justify (i), we see that $\phi_{1}=-\sum_{K}(-1)^{f(K)} a_{K} d x_{K-n}$, where the sum is only over those $K$ such that $n \in K, 2 n \in K$, and where $f(K)$ is the index of the position of $n$ in $K$. Let a subscript 0 mean that only the matrix $\mathfrak{M}_{0}$ is involved, so that $n, 2 n \in I_{0}$, and the sign of $\left\{I_{0}, K_{0}\right\}_{0}$ is to be computed from its position in $\mathfrak{M}_{0}$, not in $\mathfrak{M}$; let $K_{0}^{\prime \prime}$ denote the complement of $K_{0}$ with respect to $1, \cdots, n-1, n+1, \cdots$, $2 n-1$. Then the induction hypothesis $(n-1, p-1, r)$ for $\phi_{1}$ $=\sum a_{K_{0}} d x_{K_{0}}$ is: for all $I_{0}$ of type $(p-1-i, i), i=(p-n)^{+}, \cdots, r-1$, we have

$$
\sum a_{K_{0}}\left\{I_{0}, K_{0}^{\prime \prime}\right\}_{0}=0 .
$$

But $K_{0}$ and $K-n$ (with the noted restrictions on $K$ ) run over exactly the same sequences of indices; so also do $I_{0}$ and $I-n$. Furthermore, if $K_{0}=K-n$, then $K_{0}^{\prime \prime}=K^{\prime}-2 n$, so the above becomes: for all $I$ of type $(p-i, i), i=(p-n)^{+}, \cdots, r-1$, and $n \in I, 2 n \in I$,

$$
-\sum_{K}(-1)^{f\left(K^{\prime}\right)} a_{K}\left\{I-n, K^{\prime}-2 n\right\}=0
$$


where in the sum, $n \in K, 2 n \notin K$.

On the other hand the left-hand side when multiplied by $\pm a_{n, 2 n}$ is just the sum $T_{I}$. Namely, only the minors $\left\{I, K^{\prime}\right\}$ for which $2 n \notin K$ contain $a_{n, 2 n}$ at all. And if $n \notin K$, the coefficient of $a_{n, 2 n}$ is always zero, as can be seen immediately on expanding $\left\{I, K^{\prime}\right\}$ by its column $n$, whose only nonzero entry is $1 / d_{n}$ in row $n$. As for one of the remaining minors $\left\{I, K^{\prime}\right\}$ for which $n \in K, 2 n \notin K$, the coefficient of $a_{n, 2 n}$ that it contributes to $T_{I}$ is seen on expanding by column $2 n$ to be just $\pm a_{K}\left\{I-n, K^{\prime}-2 n\right\}$ with a sign independent of $K$, coming from the position of $a_{n, 2 n}$ in $\left\{I, K^{\prime}\right\}$, which can be ignored. Since now

$$
\left\{I-n, K^{\prime}-2 n\right\}= \pm\left\{I-n, K^{\prime}-2 n\right\}_{0}(-1)^{f(K)}
$$

where again the ambiguous sign is independent of $K$, the result follows by a summation on $K$.

The justification of (ii) is similar. On the one hand, $\phi_{2}$ $= \pm \sum a_{K} d x_{K-2 n}$, where $n \notin K, 2 n \in K$; the corresponding induction hypothesis $(n-1, p-1, r)$ is expressible as

$$
\sum a_{K}\left\{I-n, K^{\prime}-n\right\}_{0}=0 .
$$

On the other hand in the sum $U_{I}$ the only minors $\left\{I, K^{\prime}\right\}$ contributing will be those for which $n \notin K, 2 n \in K$; for clearly, $K^{\prime}$ must not contain column $2 n$, but also it must contain column $n$ with its sole nonzero entry $1 / d_{n}$, since that is the only way row $n$ can be killed off. Expanding such a minor $\left\{I, K^{\prime}\right\}$ by column $n$, we get after taking into account both the position of $1 / d_{n}$ in the minor and the subscript 0 on the brackets

$$
\pm \frac{1}{d_{n}}\left\{I-n, K^{\prime}-n\right\}_{0},
$$

so that the result again follows by summation on $K$.

PART B. Combining (1) and (3) above, we have now

$$
\sum a_{K} d x_{K}=\omega^{n-p+r} \psi+\omega_{n} \phi_{3}+\phi_{4} .
$$

Since $\sum a_{K} d x_{K}$ satisfies by assumption the hypothesis of the theorem, and since $\omega^{n-p+r} \psi$ does so also by virtue of the truth of the converse of the theorem, so must the form $\omega_{n} \phi_{3}+\phi_{4}$, so that we can work now just with this last form. We will use the induction assumption $(n-1, p, r)$ on it to show that

$$
\omega_{n} \phi_{3}=\omega^{n-p+r} \chi+\chi_{0}
$$

where $\chi_{0}$ involves neither $d x_{n}$ nor $d x_{2 n}$.

In fact the hypothesis is again (4), except that the sum is now only 
over those $K$ which contain either both $n$ and $2 n$ or neither of them. Choose any $I$ which contains neither $n$ nor $2 n$; this means that we are for the moment excluding from consideration the case $(n, p$, $p-n+1), p \geqq n$, for which $r-1=(p-n)^{+}$and for which therefore no such $I$ is admissible. Then in the sum, all the minors $\left\{I, K^{\prime}\right\}=0$ whenever $n, 2 n \notin K$ because they have a column $n$ consisting entirely of zeroes. As for the others, we have

$$
\left\{I, K^{\prime}\right\}= \pm(-1)^{f(K)}\left\{I, K^{\prime}\right\}_{0}, \quad n, 2 n \in I, K^{\prime},
$$

where the sign is independent of $K$, so that the sum is

$$
\pm \sum(-1)^{f(K)} a_{K}\left\{I, K^{\prime}\right\}_{0}=0 .
$$

But this is precisely the hypothesis of $(n-1, p, r)$, applied to the $2(n-1)-p$ form $\phi_{3}$, since $\phi_{3}= \pm \sum(-1)^{f(K)} a_{K} d x_{K-n-2 n}$, summed over $n, 2 n \in K$.

By the induction assumption, therefore, we have $\phi_{3}=\omega_{0}^{n-p+r-1} \rho_{0}$ where $\rho_{0}$ contains no $d x_{n}$ or $d x_{2 n}$. Combining this with (2) gives us (6).

For the excluded case $(n, p, p-n+1), p \geqq n$, we have $n-p+r=1$, and this gives us immediately $d_{n} \omega_{n} \phi_{3}=\omega \phi_{3}-\omega_{0} \phi_{3}$, which is (6) again, so that we can still think of it as implied by the vacuously true proposition $(n-1, p, p-n+1)$.

From (5) and (6) we have now

$$
\sum a_{K} d x_{K}=\omega^{n-p+r}(\chi+\psi)+\psi_{0}
$$

where $\psi_{0}$ is a $2 n-p$ form without $d x_{n}$ or $d x_{2 n}$ which satisfies the hypothesis of the theorem (since the other two terms of (7) do). Explicitly,

$$
\psi_{0}=\sum b_{K} d x_{K} \text { with } \sum b_{K}\left\{I, K^{\prime}\right\}=0,
$$

where $n, 2 n \notin K$ and $I$ is anything of the usual type. Excluding for the moment from consideration the case $p=2,(p=1$ is trivial), let $n \in I, 2 n \notin I$, and select any $j \in I$ different from $n$. The coefficient of $a_{j, 2 n}$ in the sum of minors must vanish; if we expand each of the minors $\left\{I, K^{\prime}\right\}$ by a double Laplace expansion using the columns $n$ and $2 n$ that they all contain and sum on $K$, the coefficient of $a_{j, 2 n}$ turns out to be

$$
\pm \frac{1}{d_{n}} \sum b_{K}\left\{I-j-n, K^{\prime}-n-2 n\right\}_{0}=0 .
$$

Here now $I-j-n$ is either of type $(p-2-i, i)$ or of type $(p-1-i$, $i-1)$ and it contains neither $n$ nor $2 n$. The above relations for different $I$ and $j$ are therefore the hypothesis of $(n-1, p-2, r)$ for the form 
$\psi_{0}$, which is of degree $2 n-p=2(n-1)-(p-2)$. We conclude therefore by the induction that $\psi_{0}=\omega_{0}^{n-p+r+1} \sigma_{0}$. Using (2) now, it may be immediately verified that

$$
\psi_{0}=\omega_{0}^{n-p+r+1} \sigma_{0}=\omega^{n-p+r}\left(\omega_{0}-(n-p+r) d_{n} \omega_{n}\right) \sigma_{0} .
$$

For the special case $p=2$, the induction is still applicable if suitably interpreted. Here the sum $\sum b_{K}\left\{I, K^{\prime}\right\}$ consists of a single term, since $K^{\prime}$ must be $(n, 2 n)$. Then $\left\{I, K^{\prime}\right\}= \pm\left(1 / d_{n}\right) a_{j, 2 n}$ where $I=(j, n)$, so the vanishing of the sum means that $b_{K}=0$, or $\psi_{0}=0$.

This completes the proof.

COROLlary. The rank of the group of homology classes represented by $p$-cycles of rank $r$ on the general abelian variety $V^{n}$ is

$$
\left(\begin{array}{c}
2 n \\
p-2 r
\end{array}\right)
$$

Proof. If $\sigma \sim E^{p-r} \cdot \tau$, then if $\sigma$ is a $p$-cycle, $\tau$ is a $(2 n-p+2 r)$ cycle. By the theorem, the homology classes in question arise by intersecting the homology group $H_{2 n-p+2 r}(V, Q)$ with $E^{p-r}$. But $H_{2 n-p+2 r}$ has rank

$$
\left(\begin{array}{c}
2 n \\
2 n-p+2 r
\end{array}\right)=\left(\begin{array}{c}
2 n \\
p-2 r
\end{array}\right)
$$

so that if we knew the intersection mapping of $H_{2 n-p+2 r}$ into $H_{p}$ had zero kernel, we would be done. Applying the Poincare duality operator, this amounts to showing $\omega^{n-p+r} \phi=0 \Rightarrow \phi=0$, if $\phi$ is a harmonic $(p-2 r)$-form.

According to Hodge's classification of harmonic forms on manifolds, [5; see 2, 4], a harmonic $q$-form has a unique representation:

$$
\psi=\sum_{i} \omega^{i} \psi_{q-2 i} ; \quad i=(q-n)^{+}, \cdots,
$$

where the $\psi_{q-2 i}$ are effective harmonic $(q-2 i)$-forms. For our $(p-2 r)$ form $\phi$, we therefore have $\phi=\sum \omega^{i} \phi_{p-2 r-2 i}, i=(p-2 r-n)^{+}, \cdots$, and so $\omega^{n-p+r} \phi=\sum \omega^{n-p+r+i} \phi_{p-2 r-2 i}=0$, by hypothesis. This last however is a $(2 n-p)$-form which represents zero; therefore by the uniqueness, all the $\phi_{p-2 r-2 i}$ must be zero, provided that it really is written in the Hodge manner, that is, provided that the power of $\omega$ which starts the sum, namely $n-p+r+(p-2 r-n)^{+}$, is not less than the power of $\omega$ which the Hodge classificatory expression starts off with, $(2 n-p-n)^{+}$ $=(n-p)^{+}$. But trivially, $n-p+r \geqq(n-p)^{+}$. Therefore all $\phi_{p-2 r-2 i}=0$ and so $\phi=0$. 


\title{
REFERENCES
}

1. H. Cartan, Fonctions theta sur le tore I, II, mimeographed seminar notes on Several Complex Variables, Paris, 1951-1952.

2. S. Chern, Complex manifolds, mimeographed lecture notes, University of Chicago, 1955-1956.

3. A. Comessatti, Sugl'indici di singolarita a piu dimensioni della varieta abeliane, Rendiconti del Seminario Matematico della Università di Padova, vol. 5 (1934) p. 50.

4. B. Eckmann and H. Guggenheimer, Formes differentielles et metrique hermitienne sans torsion, Comptes Rendus vol. 229 (1949) pp. 464-466, 489-491.

5. W. V. D. Hodge, Harmonic integrals, Cambridge, 1952, $\S 43,51$.

Massachusetts Institute of Technology

\section{CUT SETS IN TOTALLY NONAPOSYNDETIC CONTINUA ${ }^{1}$}

\author{
EDWARD E. GRACE
}

For metric spaces, F. Burton Jones has shown [1, Theorem 12] that if $D$ is an open subset of a compact continuum $M$ such that $M$ is nonaposyndetic at every point of $D$, then $D$ contains a point $x$ and $M$ contains points $y$ and $z$ such that $y$ cuts $x$ from $z$ in $M$. This paper extends the results of Jones to a certain class of topological spaces, gives a cut set in some cases where there is no cut points and gives stronger cutting properties.

Definition. ${ }^{2}$ If $A$ and $B$ are two mutually exclusive subsets of a topological continuum (i.e., a connected topological space) $T$ and there does not exist a continuum $T^{\prime}$ and an open subset $U$ of $T$ such that $[T-A] \supset T^{\prime} \supset U \supset B$, then $T$ is nonaposyndetic at $B$ with respect to $A$.

Definition. If $T$ is a topological continuum, $A$ is a subset of $T$ and $G$ is a collection of subsets of $T$ and if, for each point $x$ of $A$, there is a member $g$ of $G$ such that $T$ is nonaposyndetic at $x$ with respect to $g$, then $T$ is totally nonaposyndetic on $A$ with respect to $G$. If $A=T$ then $T$ is totally nonaposyndetic with respect to $G$. If, in addition, $G=\{\{x\} \mid x$ is in $T\}$ then $T$ is totally nonaposyndetic.

Definition. If $T$ is a topological continuum, $Z$ is the smallest cardinal number of a topological basis for $T$ and the subset $A$ of $T$

Presented to the Society, August 29, 1957; received by the editors June 25, 1957.

1 This paper is a generalization of part of a dissertation submitted to the Graduate School of the University of North Carolina in partial fulfillment of the requirements for the Ph.D. degree. The author wishes to express his appreciation to Professor F. Burton Jones for valuable suggestions.

2 The first two definitions are generalizations of definitions due to Jones [1]. Terms not defined herein are used as in [1] or [3]. 